\title{
Differential brain shrinkage over 6 months shows limited association with cognitive practice
}

\author{
Naftali Raz ${ }^{\mathrm{a}, *}$, Florian Schmiedek ${ }^{\mathrm{b}}$, Karen M. Rodrigue ${ }^{\mathrm{c}}$, Kristen M. Kennedy ${ }^{\mathrm{c}}$, Ulman Lindenberger ${ }^{\mathrm{d}}$, \\ Martin Lövdén ${ }^{\mathrm{e}}$ \\ a Institute of Gerontology and Department of Psychology, Wayne State University, Detroit, MI, USA \\ ${ }^{\mathrm{b}}$ German Center for International Educational Research (DIPF), Frankfurt am Main, Germany \\ ${ }^{\mathrm{c}}$ Center for Vital Longevity, University of Texas at Dallas, Dallas, TX, USA \\ ${ }^{\mathrm{d}}$ Center for Lifespan Psychology, Max Planck Institute for Human Development, Berlin, Germany \\ ${ }^{\mathrm{e}}$ Aging Research Center, Karolinska Institutet and Stockholm University, Stockholm, Sweden
}

\section{A R T I C L E I N F O}

Article history:

Accepted 5 April 2013

Available online 11 May 2013

\section{Keywords:}

Aging

Cerebellum

Cognitive training

Plasticity

Vascular risk

Longitudinal

MRI

\begin{abstract}
A B S T R A C T
The brain shrinks with age, but the timing of this process and the extent of its malleability are unclear. We measured changes in regional brain volumes in younger (age 20-31) and older (age 65-80) adults twice over a 6 months period, and examined the association between changes in volume, history of hypertension, and cognitive training. Between two MRI scans, 49 participants underwent intensive practice in three cognitive domains for 100 consecutive days, whereas 23 control group members performed no laboratory cognitive tasks. Regional volumes of seven brain structures were measured manually and adjusted for intracranial volume. We observed significant mean shrinkage in the lateral prefrontal cortex, the hippocampus, the caudate nucleus, and the cerebellum, but no reliable mean change of the prefrontal white matter, orbital-frontal cortex, and the primary visual cortex. Individual differences in change were reliable in all regions. History of hypertension was associated with greater cerebellar shrinkage. The cerebellum was the only region in which significantly reduced shrinkage was apparent in the experimental group after completion of cognitive training. Thus, in healthy adults, differential brain shrinkage can be observed in a narrow time window, vascular risk may aggravate it, and intensive cognitive activity may have a limited effect on it.
\end{abstract}

(c) 2013 Elsevier Inc. All rights reserved.

\section{Introduction}

Aging is accompanied by profound changes in brain structure and function, but the pace of change varies significantly across brain regions and is characterized by substantial individual differences (for reviews see Kemper 1994; Hedden and Gabrieli 2004; Raz \& Rodrigue, 2006). Reliable volume reduction in multiple cortical, subcortical, and cerebellar regions as well as thinning of the cerebral cortex have been observed in vivo in many samples, with various analytical tools, and on different imaging platforms (Fjell et al., 2009; Pfefferbaum, Sullivan, Rosenbloom, Mathalon, \& Lim, 1998; Raz, Ghisletta, Rodrigue, Kennedy, \& Lindenberger, 2010; Raz, Rodrigue, Kennedy, Dahle, et al., 2003; Raz, Rodrigue, Kennedy, Head, et al., 2003; Raz et al., 2004, 2005; Resnick, Pham, Kraut, Zon-

Abbreviations: HC, hippocampus; DLPFC, dorsolateral prefrontal cortex; OF, orbitofrontal cortex; VC, primary visual (pericalcarine) cortex; $C D$, caudate nucleus; $\mathrm{CB}$, cerebellum; FW, prefrontal white matter.

* Corresponding author. Address: 87 E. Ferry Street, 226 Knapp Building, Detroit, MI 48202, USA.

E-mail address: nraz@wayne.edu (N. Raz). derman, \& Davatzikos 2003; Scahill Fjell et al., 2003). In healthy individuals, regional changes in brain volume have been detected within time spans from five (Driscoll et al., 2009; Pfefferbaum et al., 1998; Raz et al., 2005; Rusinek et al., 2003) to less than two (Fjell et al., 2009; Raz et al., 2010) years to 6 months (Murphy et al., 2010), and in the hippocampus, reliable shrinkage was noted within a span of 4 months (Lövdén et al., 2012). However, the localization and extent of regional change vary across studies and even across different samples studied in the same laboratory (e.g., Raz et al., 2005 vs. Raz et al., 2010). Moreover, the samples in which the changes were observed cover different parts of the adult age span and vary in the mean age by more than two decades (e.g., from 52 years in Raz et al., 2005 to 76 years in Murphy et al., 2010). Notably, changes in brain structure are not limited to older adulthood. Shrinkage of at least some brain regions (e.g., HC) has been observed in younger adults (Delisi et al., 1997; Lövdén et al., 2012; Raz et al., 2005; Scahill et al., 2003), although not at the same rate as noted after the sixth decade of life (Raz et al., 2005).

Age-related shrinkage of the brain is not benign, and persons who evidence significant reduction in local brain volumes display reduced cognitive performance (Rodrigue and Raz, 2004; Raz et al., 
2008) and run increased risk of developing dementia (Murphy et al., 2010). Because brain shrinkage and its effects on cognition vary substantially among individuals, it is important to determine the factors that affect the rate of decline. An example of such moderator of brain decline is arterial hypertension (Salerno et al., 1992; Strassburger et al., 1997), a common vascular risk factor that increases the rate and widens the spread of shrinkage across the brain (Raz, Rodrigue, Kennedy, \& Acker, 2007; Raz et al., 2005, 2008).

Once age-related shrinkage of the brain is observed, it is only natural to ask what can be done about it. To date, several interventions aimed at slowing age-related brain change yielded mixed results. Whereas treating accelerators of brain aging, such as hypertension, does not slow the rate of decline (Jennings et al., 2012), other interventions such as aerobic exercise (Erickson et al., 2011) inspire cautious optimism regarding the malleability of the aging brain. One increasingly popular approach to combatting age-related brain shrinkage is perceptual-motor and cognitive training. Intensive practice on perceptual-motor and cognitive tasks of a wide range of complexity may result in significant enlargement of circumscribed brain regions and changes in white matter diffusion properties in young adults (Draganski et al., 2004, Draganski, Gaser, Kempermann, Kuhn, Winkler \& Büchel 2006; Mackey, Whitaker, \& Bunge, 2012; Schmidt-Wilcke, Rosengarth, Luerding, Bogdahn, \& Greenlee, 2010; Takeuchi et al., 2010). Most important, older brains show practice-related improvements in gray matter volume (Boyke, Driemeyer, Gaser, Büchel, \& May, 2008; Lövdén et al., 2012) and regional white matter integrity (Lövdén, Bäckman, Lindenberger, Schaefer, \& Schmiedek, 2010) as well, albeit not always and not to the same extent as the younger brains do (e.g., Wenger et al., 2012).

These findings suggest that intensive and systematic cognitive training may slow down the advancement of brain aging. However, even in younger adults the evidence of experience-induced change is far from overwhelming and significant methodological problems persist to dampen the enthusiasm about the findings. According to a recent review, $80 \%$ of extant studies of experience-dependent changes in brain structure relied on voxel-based morphometry (VBM) and a minority use other semi-automated approaches such as FreeSurfer (Thomas \& Baker, 2012). Systematic comparisons between VBM and manual morphology (Allen, Bruss, Mehta, Grbowski, Brown \& Damsio, 2008; Kennedy et al., 2009) provide ample basis for caution in interpreting differences between groups and conditions revealed by VBM. On the other hand, hypothesis-driven intervention studies with manual measurements are rare and thus far have been limited to investigation of one or two regions of interest (ROIs, e.g., Lövdén et al., 2012). In general, the hypothesis-free approach taken by the vast majority of the extant studies is problematic, especially when only small clusters of voxels that showed longitudinal change are compared between the training group and the controls (Thomas \& Baker, 2012). Notably, among the extant studies of structural plasticity, only two (Lövdén et al., 2012; Wenger et al., 2012), conducted on the same sample, compared practice-related changes in younger and older adults, and most have not evaluated the critically important group $\times$ time interaction (Thomas \& Baker, 2012). In addition, with a couple of notable exceptions, intervention studies of structural change relied on small samples, with a median size of 38 and a range of 11-120 participants (Thomas \& Baker, 2012, Table 1). In summary, Thomas and Baker (2012) concluded that in studies of experience-based change in brain structure, the effects are small, highly localized, poorly replicated, transient, and restricted to younger adults.

In this study, we addressed four questions, while trying to take into account threats to validity outlined by previous studies and summarized in Thomas and Baker (2012). First, we inquired whether differential age-related shrinkage in healthy young and old adults happens fast enough to be noticed within a 6-month time window. In pursuit of that objective, we selected regions that have been shown to change in relatively short periods (the prefrontal cortex, the hippocampus, the cerebellum, and the caudate nucleus) and a control region that evidenced no significant shrinkage in previous longitudinal studies - the primary visual cortex. Second, we examined if individuals reliably differ in their rates of brain shrinkage, even when observed over relatively short intervals. The third question was whether vascular risk moderates age-related declines in brain volume. Based on the reviewed evidence, we expected vascular risk to exacerbate shrinkage of the gray matter in the prefrontal cortex and the hippocampus. The fourth objective was to evaluate whether persons who undergo intensive cognitive intervention show lesser regional brain shrinkage than their counterparts who do not participate in the program. We addressed these questions by examining short-term changes in the regional brain volumes of healthy adults who participated in intensive training of multiple cognitive skills. We hypothesized that healthy adults, especially the older among them, would show significant shrinkage within 6 months at least in the brain regions with established vulnerability, such as the hippocampus, the prefrontal cortex, and the cerebellum. We expected to observe significant individual differences in the rate of change, and we hypothesized that persons with hypertension would show greater shrinkage than their normotensive peers would. Finally, we expected that the brains of people who underwent intensive cognitive practice would evidence lesser shrinkage than those of the control group participants. We expected to observe the benefits of practice in the regions with known associations to higher cognitive activities that are tapped by the cognitive tasks employed in this study: episodic memory, working memory and perceptual speed. Thus we selected the hippocampus, in which extensive training related to high memory load is believed to induce functional, metabolic and structural changes (Groussard et al., 2010; Maguire et al., 2000; Mårtensson et al., 2012; Roche et al., 2009), the prefrontal cortex that showed changes after memory, attention and working memory training (Engvig et al., 2010; Hoekzema et al., 2011; Mårtensson et al., 2012), and the cerebellum which evidenced structural growth after working memory training (Hoekzema et al., 2011).

Table 1

Longitudinal change in regional cerebral volumes: a summary of the univariate latent change models

\begin{tabular}{|c|c|c|c|c|c|}
\hline ROI & Baseline mean & $\begin{array}{l}\text { Mean change } \\
\text { baseline - follow-up }\end{array}$ & Baseline, variance & $\begin{array}{l}\text { Variance of change } \\
\text { baseline - follow-up }\end{array}$ & Annual\% change \\
\hline Hippocampus & 3551 & $-50^{*}$ & $170,709^{*}$ & $15,048^{*}$ & -1.42 \\
\hline Lateral prefrontal cortex & 10,569 & $-104^{*}$ & $3,813,815^{*}$ & $77,429^{*}$ & -2.51 \\
\hline Orbital frontal cortex & 4924 & -33 & $1,031,243^{*}$ & $44,333^{*}$ & -0.83 \\
\hline Primary visual cortex & 3026 & -7 & $200,748^{*}$ & $5195^{*}$ & -0.48 \\
\hline Caudate & 4160 & $-39^{*}$ & $412,269^{*}$ & $7988^{*}$ & -1.21 \\
\hline Cerebellum & 59,703 & $-494^{*}$ & $40,457,233^{*}$ & $650,285^{*}$ & -1.86 \\
\hline prefrontal white & 19,656 & 80 & $10,247,462^{*}$ & $433,687^{*}$ & 1.28 \\
\hline
\end{tabular}

Notes: All volumes are in $\mathrm{mm}^{3}$, adjusted for intracranial size. 


\section{Method}

\subsection{Participants}

The participants were paid volunteers, residents of Berlin, Germany, who responded to advertisement in newspapers, word-ofmouth recommendations, and fliers that invited them to join a study on the effects of intensive cognitive training on brain and behavior (the COGITO study; see Schmiedek, Lövdén, \& Lindenberger, 2010, for details). The main study involved 101 younger (aged 20-31 years) and 103 older adults (aged 65-80 years). These individuals took part in an intensive 100-days program of cognitive training, during which they practiced several tasks covering three domains of cognition: working memory (three tasks), episodic memory (three tasks), and perceptual speed (six tasks; see Schmiedek et al., 2010 for details). Out of this sample, we recruited for the MRI study individuals with no history of psychiatric, neurological, metabolic, cardiovascular or endocrine disease. Some participants reported diagnosis of essential hypertension and were taking anti-hypertensive medications. MR imaging was performed before and after the cognitive training intervention, with the delays between scans ranging from 135 to 232 days. There were no differences in delay between the scans by age group, sex, hypertension diagnosis or training assignment: all $F<1$. All measures were tested for association with the delay, and none were found. Therefore, delay was not included in the statistical models. A no-contact control group met the same exclusion and inclusion criteria.

The sample of this study consisted of 35 younger (age $25.27 \pm 3.18$ ) and 37 older (age $70.88 \pm 3.82$ ) adults. The age groups did not differ in sex composition (61\% vs. $53 \%$ men among the younger and the older participants, respectively; $\chi^{2}=.452$, $p=.501)$ or inter-scan delay $(t=.145, p=.885)$. Twenty-five younger and 24 older participants formed the training group, whereas 10 younger and 13 older adults constituted a no-contact control group. The control group that was originally selected in the COGITO study (see Schmiedek et al., 2010) was made unavailable to this study because of MRI equipment problems. We therefore recruited a new control group from the same populations using the same advertising methods and the same exclusion and inclusion criteria.

The experimental and control groups did not differ in age, $t(67)=.808, p=.420$, delay between the MRI scans, $t=1.07$, $p=.290$, or sex composition, $\chi^{2}=.973, p=.323 ; 53 \%$ men and $65 \%$ men in the experimental and control group, respectively. There were two notable differences between the groups. First, eight of the training group participants but none of the controls had hypertension, $\chi^{2}=4.450, p=.044$. Second, whereas, as expected, younger participants performed better than their older counterparts: $(66.13 \pm 1.42$ vs. $46.63 \pm 1.34, \quad F(1,65)=99.76$, $p<.00001)$, the control group participants performed better than the experimental group: $60.71 \pm 1.60$ vs. $52.04 \pm 1.12$; $F(1,65)=19.69, p=.00004$. As indicated by Age $\times$ Training Group interaction $(F(1,65)=4.36, p=.040)$, this difference was mainly due to substantially better performance of younger controls compared to the younger experimental group participants: $72.20 \pm 2.40$ vs. $59.76 \pm 1.52$; Tukey HSD test $p=.00018$. The difference between the older controls and older experimental group participants was not-significant: $48.92 \pm 2.11$ vs. $44.33 \pm 1.67$, Tukey $p=.32$, ns. Older participants attained higher scores on the wordknowledge test than the younger participants did: $F(1,65)=43.13, p<.00001$. No difference between the control and experimental groups were observed on the vocabulary test $(F<1)$. Thus, controls happened to be somewhat better on at least two important indicators of physical and cognitive health than the experimental group participants were.

\subsection{Cognitive training protocol}

Participants practiced daily for $1 \mathrm{~h}$ on individual computerized testing stations, up to six participants per lab room, for an average of 100.89 (92-109) sessions distributed over the period between pre and posttest. The cognitive training battery consisted of three working memory, three episodic memory, and six perceptual speed tasks. Individual sessions occurred on up to 6 days a week. At the end of each session, participants received feedback on their performance on all tasks, including average accuracies and reaction times. For a detailed description of the tasks, see Schmiedek et al., 2010.

\subsubsection{Pretest-posttest assessment}

Participants underwent 10 days of behavioral pre-testing in group sessions that lasted 2-2.5 h. Measurements consisted of self-report questionnaires, cognitive tasks included in the daily phase, and transfer tasks (for a detailed description, see Schmiedek et al., 2010). Ten posttest group sessions (1.5-2 h each) consisted of re-administration of the pre-test cognitive tasks and additional self-report measures. In this study, we measured cognitive performance at baseline and follow-up in three major cognitive domains: working memory (three index tasks), episodic memory (three index tasks), and perceptual speed (six index tasks). All tasks were administered during the daily training phase of the study.

\subsection{MRI protocol}

\subsubsection{Acquisition}

A pre-test brain-imaging session was conducted after the behavioral pretest and immediately before the daily assessment phase; the post-test images were acquired shortly after the completion of the behavioral posttest. The images were acquired at both occasions on the same GE Signa LX 1.5 Tesla system (General Electric, Milwaukee, WI) with actively shielded magnetic field gradients (maximum amplitude $40 \mathrm{mT} \mathrm{m}^{-1}$ ). The MR protocol included a T1-weighted sagittal 3D scan (contrast-optimized spoiled gradient-echo sequence, 124 slices, slice thickness = $1.5 \mathrm{~mm}$, FOV $250 \times 250 \mathrm{~mm}^{2} ; 256 \times 256$ matrix; TE = $8 \mathrm{~ms}$; $\mathrm{TR}=24 \mathrm{~ms}$; flip angle $=30^{\circ}$ ).

\subsubsection{Image processing and manual volumetry}

Image processing and regional volumetry are described in detail elsewhere (Raz et al., 2004, 2005, 2010). The baseline and followup images were coded, mixed, and assigned randomly to two tracers. The tracers were blind to the time of acquisition and to the demographic characteristics of the participants. Reliability of all manual ROI measures assessed by a conservative index, intraclass correlation for random raters (ICC [2], Shrout and Fleiss, 1979), exceeded .93. The volumes of the intracranial vault (ICV) and seven regions of interest (ROI) - lateral prefrontal cortex (LPFC), orbital frontal cortex (OFC), the adjacent prefrontal white matter (PFw), primary visual (calcarine) cortex (VC), the hippocampus (HC), the caudate nucleus $(\mathrm{Cd})$, and the cerebellar hemispheres $(\mathrm{Cb})$ - were computed from measured areas (see Raz, Rodrigue, Kennedy, Dahle, et al. 2003; Raz, Rodrigue, Kennedy, Head, et al., 2003; and Raz et al., 2004 for details).

As expected, men had significantly greater ICV than women did, $F(1,62)=41.069, p<.00001$, but we observed no sex $\times$ ICV interactions for any of the baseline regional volumes ( $F$ s ranging from .12 to 1.67 , all ns) or at follow-up (all $F$ s $<1$ ). The ICV at follow-up was significantly larger than at baseline: a small but consistent difference of $.47 \%$. Moreover, the ICV volume increase was smaller for the control group (.26\%) than for the experimental group (.85\%), $t(70)=2.836, p=.006$. The correlation over time between the ICV 
measures was $r=.990, p<.001$. The sources of the observed ICV differences are unclear. It may arise from changes in head positioning, gradients, RF, and participant's hydration level, to name a few possibilities. The assumption is that all these factors affect everything that is imaged in the scanner inside a given cranium. Incidentally, other studies reported longitudinal ICV change. In one study we found a $0.3 \%$ increase in ICV between baseline and the first retest but not between the first and the second retest after the same delay (Lövdén et al., 2012). In another study, we observed a similar small difference in the opposite direction, i.e. a reduction of .3\% (Raz et al., 2005). In the present study, there was no interaction between ICV and training group membership or age at any measurement occasions for any of the ROI volumes: all $F<1$. We could therefore adjust for individual and sex differences in body size, as well as variations in MRI scanning across two occasions, by adjusted all regional volumes for the ICV through a regression-ANCOVA approach described in previous publications (Jack et al., 1989; Raz et al., 2004).

\subsection{Statistical analyses}

To improve reliability of the cognitive measures, we computed unit-weighted composites of performance on the training tasks in each cognitive domain. Each measure was first $z$-transformed using the pretest means and standard deviations of the total sample, so that age and time differences were preserved. Next, the measures of working memory (three tasks), episodic memory (three tasks), and perceptual speed (six tasks) were averaged to form the three cognitive domain composites. Because the cognitive data were available only for the intervention group, we analyzed performance for that group with a series of univariate 2 (time; pretest vs. posttest) $\times 2$ (age group; young vs. old) mixed analyses of variance (ANOVAs) for each cognitive composite.

To evaluate the change in regional brain volumes between two occasions, we used the latent difference score (LDS) model. This structural equation modeling technique is well suited for evaluating individual differences in change with two-occasion data (McArdle, 2009; McArdle \& Nesselroade, 1994; Rogosa \& Willett, 1985), and has been used in previous investigations of brain aging (McArdle et al., 2004; Raz et al., 2005; Raz et al., 2008, 2010). We used LDS models to generate latent factors for each ROI at pretest and posttest using left and right volumes as indicator variables. Change between measurement occasions is included in the model by regressing the posttest factor on the pretest factor with a fixed regression weight of one and an additional latent change factor on which the posttest factor has a fixed loading of one. Intercept and residual variance of the posttest factor are fixed at zero. Factor loadings, intercepts, and residual variances of the indicator variables are constrained as equal across pretest and posttest (for details, see Raz et al., 2005). Thus, the mean and the variance of the latent change factor represent average change and individual differences in change, respectively, at the level of latent factors. Both parameters can be tested for significance with likelihood ratio tests, using comparisons to models in which these parameters are fixed to zero. A major advantage of the LDS approach is that it enables assessment of mean change and individual differences in change separately from measurement error, such as unsystematic sources of inaccuracy in volume tracing. In addition, demonstrating metric invariance of the measurement models across time, as was the case in this study, guarantees that the regions of interest are represented in the same way at both measurement occasions, and thus further enhances the validity of the results.

We tested separate LDS models for each ROI in a two-step process. First, for each region, we fitted a model that contained volume of that region at baseline and follow up, without predictors of level and change in the model. All these models fit the data reasonably well, all $\chi^{2} \mathrm{~s}<5.82, d f s=3, p s>.121$, CFIs $>.995$, and RMSEAs $<.113$. The $95 \%$ confidence intervals of the RMSEA included .05 in all cases. At the second step, we evaluated the effects of age at baseline, sex, training group, and hypertension by conducting multiple regressions of the latent factors of level and change in brain volume on the corresponding independent variable. For tests of statistical significance, in the second step, we used Bonferroni corrections for multiple comparisons.

\section{Results}

\subsection{Changes in regional brain volumes}

\subsubsection{Latent difference scores models}

Table 1 summarizes the univariate LDS models for change in regional cerebral volumes between the baseline assessment and the follow-up. The results of these analyses indicate that the mean volumes of four out of seven examined regions shrunk within less than 6 months. Significant mean shrinkage occurred in the hippocampus, the lateral prefrontal cortex, the caudate nucleus, and the cerebellum. Notably, all seven measured ROIs evidenced significant variance in change, including those that showed no mean change. To illustrate the mean change in volume, we computed annualized percentage change (APC) index by subtracting the baseline volume from the follow-up value, dividing the result by the baseline volume, multiplying by 100 and dividing by the delay duration in months (i.e., fraction of years). The APC is an imperfect index of change. It was computed on raw scores, and its limitations include measurement errors and the fact that it does not take into account variability. The APCs for all regions also appear in Table 1.

\subsubsection{Age, sex, training, and hypertension effect on volume change}

The results of step 2 analyses, at which age at baseline, sex, training status, and hypertension entered the LDS model as predictors of level and change in brain volume appear in Table 2 . The effects of age on volume (age-related differences) were significant for all examined structures, with the greatest advantage of the younger participants in the volume of the prefrontal cortex and the smallest in the volume of the primary visual cortex. Men had a significantly greater volume of prefrontal white matter even after adjustment for intracranial volume. However, there were no sex differences in

\section{Table 2}

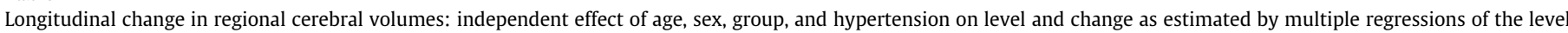
and change factors on these variables.

\begin{tabular}{|c|c|c|c|c|c|c|c|c|c|c|c|c|c|c|c|}
\hline & & \multicolumn{2}{|l|}{$\mathrm{HC}$} & \multicolumn{2}{|c|}{ DLPFC } & \multicolumn{2}{|l|}{ OF } & \multicolumn{2}{|l|}{ VC } & \multicolumn{2}{|l|}{$\mathrm{CD}$} & \multicolumn{2}{|l|}{$\mathrm{CB}$} & \multicolumn{2}{|l|}{ FW } \\
\hline & & $\mathrm{L}$ & $\mathrm{C}$ & $\mathrm{L}$ & $\mathrm{C}$ & $\mathrm{L}$ & $\mathrm{C}$ & $\mathrm{L}$ & C & $\mathrm{L}$ & $\mathrm{C}$ & $\mathrm{L}$ & $\mathrm{C}$ & $\mathrm{L}$ & C \\
\hline Age & $-.64^{*}$ & -.33 & $-.76^{*}$ & -.02 & $-.80^{*}$ & .31 & $-.52^{*}$ & .11 & $-.77^{*}$ & .22 & $-.63^{*}$ & .02 & $-.67^{*}$ & -.05 & \\
\hline Sex & -.22 & .00 & -.16 & -.10 & -.04 & -.03 & -.08 & .06 & $-.19^{*}$ & .15 & $-.21^{*}$ & .26 & $-.32^{*}$ & -.06 & \\
\hline Group & -.24 & .06 & -.08 & .07 & -.07 & -.26 & .01 & -.19 & .04 & -.06 & -.11 & $-.40^{*}$ & -.09 & .02 & \\
\hline Hypertension & .09 & -.10 & -.10 & -.09 & -.08 & -.36 & .00 & -.43 & -.03 & -.11 & -.04 & $-.34^{*}$ & -.09 & .01 & \\
\hline
\end{tabular}

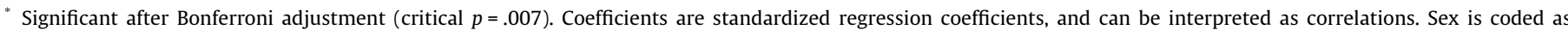

$1=$ man and $2=$ women. Group $1=$ experimental and $2=$ control; Hypertension $0=$ normotensive and $1=$ hypertensive; $\mathrm{L}-$ level, $\mathrm{C}-$ change. 
change. The diagnosis of hypertension had significant influence on change only in the cerebellum. The effect of training (Group) was significant only for the cerebellum (see Fig. 1 for individual change trajectories, and training-related differences).

In the intervention group, cognitive performance (see Table 3) improved over time in each measured domain: working memory, $F(1,47)=254.23, p<0.001$, episodic memory, $F(1,47)=99.37$, $p<0.001$, and perceptual speed, $F(1,47)=141.98, p<0.001$. Younger adults had higher scores on all cognitive composites: working memory, $F(1,47)=35.87, p<0.001$, episodic memory, $F(1,47)=$ 47.76, $p<0.001$, and perceptual speed, $F(1,47)=84.20, p<0.001$.
The age group $\times$ time interaction reached significance for perceptual speed, $F(1,47)=41.17, p<0.001$, and episodic memory, $F(1,47)=24.01, p<0.001$, indicating larger increases over time for younger relative to older adults. No other effects were significant.

To assess the specificity of training effect on changes in the cerebellar volume, we examined the correlations between individual differences in volume change and changes in performance on specific tasks among the experimental group participants. We modeled the pre- and post-test latent factors in each cognitive domain (working memory, episodic memory, and perceptual speed) with the LDS model, in a similar way as we did with the
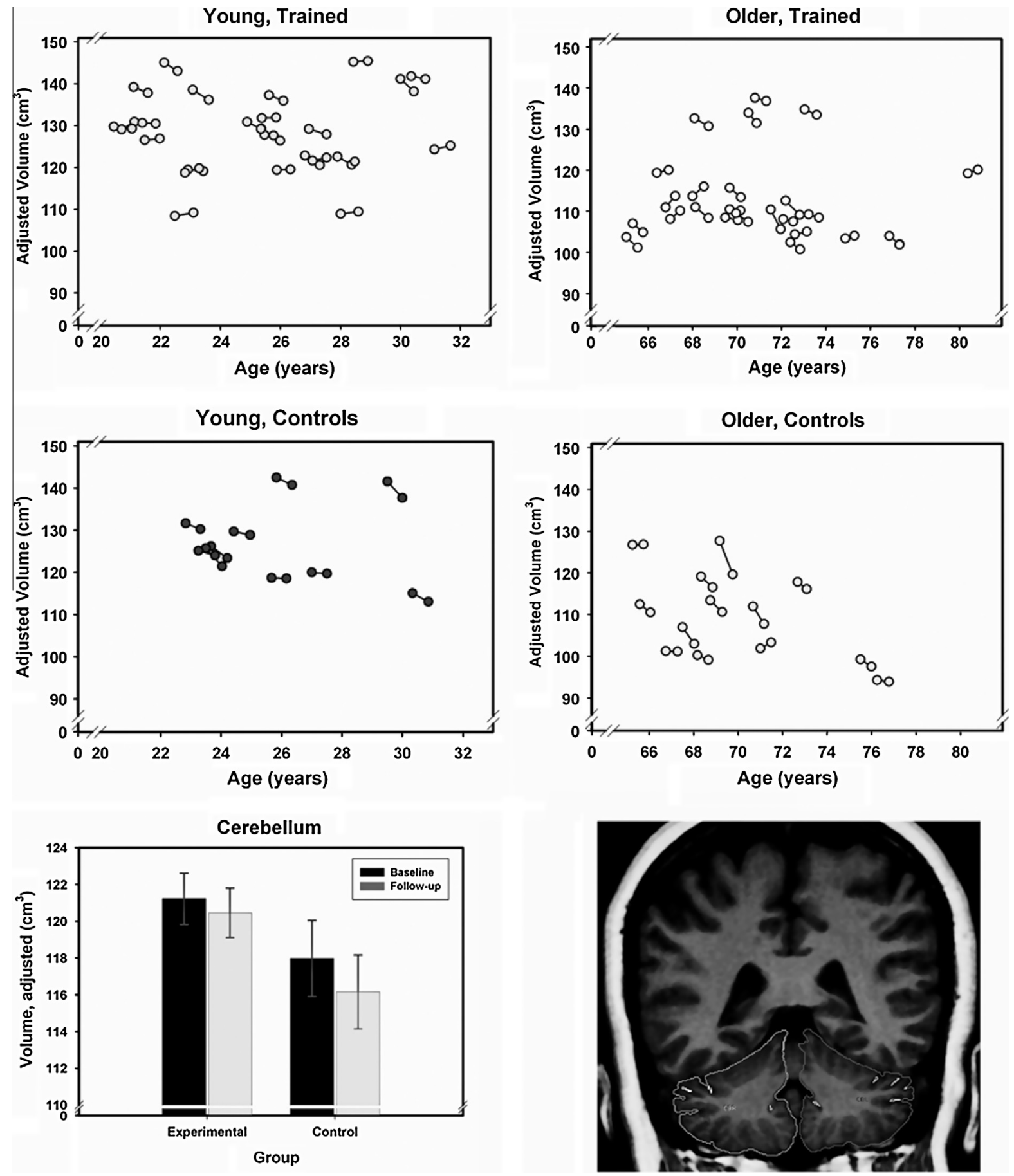

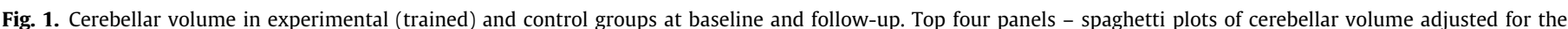

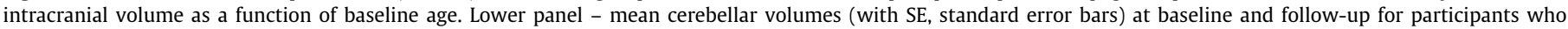
underwent cognitive training and controls. Volumes of cerebellar hemispheres include the cortex, the white matter, and the deep nuclei were traced as shown. 
Table 3

Cognitive performance before and after practice.

\begin{tabular}{|c|c|c|c|c|c|c|c|c|}
\hline \multirow[t]{3}{*}{ Cognitive Domain } & \multicolumn{4}{|l|}{ Young } & \multicolumn{4}{|l|}{ Old } \\
\hline & \multicolumn{2}{|c|}{ Pretest } & \multicolumn{2}{|c|}{ Posttest } & \multicolumn{2}{|l|}{ Pretest } & \multicolumn{2}{|c|}{ Posttest } \\
\hline & Mean & SD & Mean & SD & Mean & SD & Mean & SD \\
\hline Working memory & 0.50 & 0.69 & 1.78 & 0.81 & -0.52 & 0.46 & 0.85 & 0.56 \\
\hline Episodic memory & 0.48 & 0.88 & 1.75 & 1.31 & -0.50 & 0.46 & -0.06 & 0.82 \\
\hline Perceptual speed & 0.54 & 0.65 & 2.11 & 0.53 & -0.56 & 0.75 & -0.10 & 0.82 \\
\hline
\end{tabular}

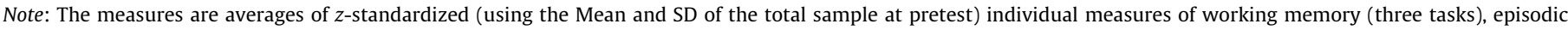
memory (three tasks), and perceptual speed (six tasks), respectively.

brain volume measures (see Schmiedek et al., 2010, for details). The relations between the cerebellum volume and changes therein to level and changes in performance were tested in separate bivariate LDS models for each cognitive domain. In none of the models did level of cognitive performance or change therein correlate with cerebellum volume (all $r s<.38$, ps $>.117$ ).

\section{Discussion}

\subsection{Neuroanatomical changes}

\subsubsection{Regional brain shrinkage - How fast?}

The results of this study show that significant shrinkage of selected brain structures occurs in healthy adults within less than 6 months. We observed mean volume changes in four out of seven examined brain regions. The pace of change in these regions was equivalent to $1.4-2.5 \%$ per annum, with the lateral prefrontal cortex exhibiting the fastest rate of shrinkage. These shrinkage rates are greater than the ones reported in a 5-year longitudinal study (Raz et al., 2005) but smaller than those found in another sample (Raz et al., 2010) evaluated with the tracing method identical to the one employed here. The sources of this heterogeneity in shrinkage await systematic study. Some portion of this heterogeneity may reflect differences among the populations from which the different samples were drawn: Memphis, Tennessee (Raz et al., 2005) vs. Detroit, Michigan (Raz et al., 2010) vs. Berlin, Germany (this sample). In addition, variations in the duration of intermeasurement intervals may contribute to differences in shrinkage rates, even when expressed as APC. A quantitative evaluation (meta-analysis) of contributions to this heterogeneity would most useful but it will have to wait for accumulation of a sufficient number of longitudinal studies.

\subsubsection{Individual differences in brain shrinkage}

The finding of individual differences in volume changes in this sample contributes to understanding the shape of brain aging trajectories. General heterochronicity of life-span trajectories of brain development and aging is well established (Lenroot and Giedd, 2006; Raz \& Kennedy, 2009). However, variability of age-related neuroanatomical change is substantial (Raz et al., 2005, 2010). Even a cursory examination of individual trajectories in this and other longitudinal studies reveals that for some individuals, the slope of change between two measurement occasions is reversed relative to the mean trajectory, and to most other individual trajectories. The nature of such reversals is unclear, as the neurobiological mechanisms of brain shrinkage observed on MRI are unclear. Gross volume reduction may reflect changes in neural number and size (Bobinski et al., 2000), loss of intralaminar myelin (Courchesne et al., 2000), rarefaction of arterioles and reduction in density of capillary networks (Riddle, Sonntag, \& Lichtenwalner, 2003), and fluctuation in hydration (Duning et al., 2005). It is also conceivable that changes in dendritic arborization and local capillary density contribute to variation in brain volumes. Regional brain volumes may be determined by multiple factors that fluctuate and counteract, thus creating a state of dynamic equilibrium that changes in a quasi-periodic fashion but shifts downwards over time.

The MRI technique used in this study is too coarse to present a true picture of such quasi-periodic process as it is incapable of tracking changes in individual cortical laminae, not to mention single neurons and dendritic branches, and it is incapable of assessing changes in density of small blood vessels in a given ROI. The Nyquist frequency of such a process is unknown but it is highly unlikely to be faithfully captured even in a relatively short (6-month) window used in this study. However, some sources of individual differences in age-related change can be discerned even with current methods. The methods that are best suited to discover neurobiologically meaningful change remain to be established. Current MRI-derived measures of volume and cortical thickness are still very coarse. Moreover, their neurobiological validity is not wellestablished. For instance, unlike manual MRI measures (Bobinski et al., 2000), VBM-derived estimates of local gray matter density do not correlate with actual neuron counts, neuronal nuclear antigen expression or presence of glial fibrillary acidic protein (Eriksson et al., 2009). Carefully performed manual volumetry, with all its limitations, is probably a preferred method of measurement, provided that it is conducted by experienced well-trained operators who attain high reliability. Significant limitations of semiautomated methods have been outlined in several studies (e.g., Kennedy et al., 2009, see Thomas \& Baker, 2012 for a review). For example, FreeSurfer produces volume estimates that overestimate the actual volume of structures such as the hippocampus by about 25\% (Cherbuin, Anstey, Réglade-Meslin, \& Sachdev, 2009) and correlate only .77-.81 with manual measures (Cherbuin et al., 2009; Shen et al., 2010), probably because of over-inclusive definition of the hippocampus in the FreeSurfer labeling. Clearly, future developments in MRI technology and software are needed to improve the resolution and validity of the methods that are relied upon in research on structural changes in healthy human brains.

\subsubsection{The role of vascular risk factors in brain aging variability}

The results of this study are in agreement with the extant reports of major vascular risk factors such as hypertension exacerbating age-related regional brain shrinkage. When using a conservative adjustment for multiple comparisons, hypertension reliably affected shrinkage rate at least in one region, the cerebellum. The finding of negative impact of hypertension on cerebellar volume is in agreement with early reports (Strassburger et al., 1997), although not with more recent longitudinal studies (e.g. Raz et al., 2005). With a less conservative decision rule, we note the same effect in the pericalcarine cortex, a region that exhibits relative stability in most of the extant investigations of the aging brain but may be sensitive to age-related increase in vascular risk (Raz \& Kennedy, 2009 for a review). We did not replicate previously observed exacerbation of hippocampal (Korf, White, Scheltens, \& Launer, 2004; Raz et al., 2005), and prefrontal (Raz et al., 
2005) shrinkage by hypertension. Given the low statistical power of this study, especially with regards to effects of hypertension, lack of replication should be interpreted with great caution. It is plausible, however, that specific targets of hypertension effects on parenchymal integrity vary by the participants' age and duration of follow-up. Longitudinal studies with greater density of measures and a larger number of hypertensive participants are needed to examine the influence of vascular risk on individual differences in brain aging.

\subsection{Neuroanatomy and cognitive performance}

\subsubsection{Attenuation of brain shrinkage by cognitive practice}

The effort to attenuate brain decline with intensive cognitive intervention showed a promise of success at least for one brain structure out of seven examined - the cerebellum. We were unable to demonstrate association of cognitive practice and change in the other brain regions, some of which (e.g., the hippocampus) play a pivotal role in support of the cognitive operations that our participants practiced for 100 days, whereas others (e.g., primary visual cortex) were selected as control locations. Although cognitive performance clearly improved after practice, especially in the younger participants, the reduced cerebellar shrinkage did not significantly relate to the initial cognitive attainment or to the success in training program. The observed benefits of cognitive training may therefore represent a general response to increase in environment complexity. In animal models, environmental manipulations of complexity produce extensive neuro-, glia-, angio- and synaptogenesis as well as dendritic and axonal proliferation (Black, Sirevaag, \& Greenough, 1987; Kleim, 2007; Black, Isaacs, Anderson, Alcantara, \& Greenough, 1990; Mora et al., 2007), and different types of environmental intervention may cause different types of structural change (Black et al., 1990; Isaacs et al., 1992; Kleim et al., 2007). At the current state of knowledge, it is impossible to determine which of these physiological and anatomical changes occurred and which ones account for the observed effect on the MRI-based measures. Note also that the lack of specific association between performance changes and brain volume should, given limited power, be carefully interpreted. Other recent findings have shown associations between brain changes and performance gains, at least in younger adults (e.g., Mårtensson et al., 2012). It is unlikely, however that training intensity played a role in our relatively isolated and unspecific findings: our participants underwent longer practice ( 100 days of about 90 min of practice) than participants in other studies that reported significant change (e.g., Engvig et al., 2010 - 50-60 days, Hoekzema et al., 2011 - 14 days).

\subsubsection{The role of cerebellum and cognitive performance}

The effect of cognitive training on the cerebellum is a novel finding and, to the best of our knowledge, there are no comparable investigations involving this region. Our results are in accord with findings of significant cellular plasticity in cerebellar neurons (Abrahamsson, Cathala, Matsui, Shigemoto, \& Digregorio, 2012), and suggest that cerebellar plasticity deserves greater attention in studies of cognitive training (cf. Diamond, 2000). Although the observed reduction in cerebellar shrinkage is unlikely to reflect a specific effect of training on one or many of the tasks, the link between cerebellar volume and these tasks is plausible. The cerebellum is a sophisticated multipurpose processing device with long-reaching connections to the motor, premotor and prefrontal association cortices (Strick, Dum, \& Fiez, 2009). It is involved in a wide range of motor, affective, and cognitive functions, all of which could have improved in the course of 100 days of training. For instance, even relatively simple working memory tasks activate lateral cerebellum (Cabeza \& Nyberg, 2000), and age differences in performance on speeded working memory tasks are associated with structural differences in the cerebellum (Eckert, Keren, Roberts, Calhoun, \& Harris, 2010). The neural substrates of cognitive skill map on cerebellar and cortical regions that are distinct from the motor circuits, for example, the lobules VII-VIII of the cerebellum (especially Crus II region) and the LPFC (Salmi et al., 2010). Unfortunately, our method lacked resolution to assess the changes in those circumscribed regions of the cerebellum. Moreover, the gross volume measures may not have been sufficiently sensitive to rapid experience-induced changes. Other indicators such as white matter diffusion properties appear to respond to learning in a much more rapid manner (Engvig et al., 2012; Landi, Baguear, \& Della-Maggiore, 2011; Lövdén, Bodammer, Kühn, et al., 2010; Sagi et al., 2012) and may undergo subtle regional changes that were missed by the method employed in this study.

\subsection{Implications for models of cognitive and brain plasticity}

The results of this study support the argument that the relationship between brain and cognition is a two-way street. The brain is important for cognitive performance but the former can also supply the capacity to respond to raising demands on the latter (Lövdén, Bäckman, Lindenberger, Schaefer, \& Schmiedek, 2010). It is interesting that the only region that showed significant change after cognitive practice was also one of the most vulnerable to aging, the cerebellum (e.g., Andersen, Gundersen, \& Pakkenberg, 2003; see Raz \& Kennedy, 2009 for a review). In a way, the "dark side of plasticity" principle (Arendt, 2003; Raz, 2000), according to which brain structures that display the highest degree of plasticity are those that are destined to show the greatest impact of aging, was turned on its head, with one of the most age-sensitive structures evidencing the greatest ability to change.

It is unclear what physiological phenomena the reported changes in volume and density of the brain tissue represent. Given significant loss of arteriolar and capillary density, alteration of their microstructure, and reduction in the pace of angiogenesis with aging (Riddle et al., 2003), it is plausible that microarchitecture of the cerebrovascular system is the primary respondent to volume gains brought by cognitive intervention. Indeed, according to some findings, this is a distinct possibility (Pereira et al., 2007), and because synaptogenesis and angiogenesis are far from independent, the contributions of vascular and neural mechanisms (Farkas \& Luiten, 2001) to experience-related plasticity may be difficult to disentangle.

To date, the research efforts aimed at understanding the neural substrates of cognitive plasticity centered exclusively on the cerebral cortex and the hippocampus (e.g., May, 2011), with the cerebellum largely ignored or assigned a role of a motor learning organ (see Ito, 2002, for a historical review). The results reported here call the attention of researchers back to the cerebellum, which for quite a while has been known as not just an organ of motor learning but a full participant in many complex cognitive processes (Botez, Botez, Elie, \& Attig, 1989). Moreover, comparison to nonhuman primates reveals a significant evolutionary development in the human cerebellum such as increase in the relative volume of Crus I and Crus II, the cerebellar compartments that project to the tertiary association areas of the prefrontal cortex (Balsters et al., 2010). In the extant literature, one can find references to changes in cerebellum after cognitive training of children with attention deficit disorder (Hoekzema et al., 2011). Our findings show that cerebellar changes accompany cognitive practice in healthy adults as well.

\subsection{Limitations}

Consideration of several limitations of this study should help in interpreting its results. First, the lack of intermittent measures of 
regional brain volumes precluded a more nuanced analysis of the volume change at various stages of training. The cerebellum may play different roles and attain a variable degree of prominence at different stages of skill acquisition. For example, activity in some cerebellar regions connected to the tertiary association areas of the cortex (e.g. area 46) increases as training of a cognitive skill progresses to automaticity (Balsters \& Ramnani, 2011) and spatial navigation learning shows different degree of dependence on hippocampus and cerebellum at different stages of information transfer (Rochefort et al., 2011). Thus, it is possible that sensitivity of cerebellar volume to training reflects the high degree of automaticity of performance after a lengthy and intensive process of acquisition. Denser measurements would have facilitated uncovering the links between volume change and cognitive improvement. A study of young adults provides an example of such investigation of the time course of practice-related neuroanatomical change (Driemeyer, Boyke, Gaser, Büchel, \& May, 2008). Having only two measurement occasions and a relatively narrow age range within age groups precluded the estimation or assessment of nonlinear change.

Second, for unknown reasons, the experimental group exhibited worse health and lower cognitive ability than the controls did. Although hypertension was related to greater shrinkage, the experimental group that included hypertensive participants showed overall lesser decline in the $\mathrm{Cb}$ volume than the control group composed of self-reported normotensives. Thus, it seems that presence of hypertension in the experimental group worked against the hypothesis of group differences. It is possible however, that presence of undetected hypertension among the control participants resulted in observed greater $\mathrm{Cb}$ shrinkage. Given that all participants came from the same pool in a community with reliable medical care, this is an unlikely scenario. Nevertheless, it is unclear if the ameliorative effect of cognitive training on cerebellar shrinkage emerged in spite the health and performance differences or it occurred because the experimental group had more room for improvement. Close matching of experimental and control group on this important parameter should be implemented in future intervention studies that include older adults.

Third, although we drew our control participants from the same population as we recruited our experimental group, we were unable due to technical problems with the scanning equipment to use the data for the originally selected control group. Thus, this study is a non-randomized controlled observational study and as such it cannot support strong claims of treatment effectiveness. As we discussed above, the differences between controls and participants of the experimental group are such that they are more likely to counter treatment success than to promote it.

Fourth, the logic of hypothesis testing allows claiming the significant impact of cognitive practice on the cerebellum; it precludes, however, a clear declaration of the lack thereof in the other regions examined in this study. This is especially true in light of the relatively low statistical power of the present study. This drawback is one of the problematic aspects of this area of research enumerated in the introduction. Despite the high cost and logistical difficulties inherent in longitudinal investigations, this limitation must be addressed in the future studies.

Fifth, the time scope of this study was relatively limited. It is unclear how long the observed gains, cognitive and neuroanatomical, will last. In studies that first reported significant structural changes in the brains of people who acquired a novel perceptual-motor skill, the neuroanatomical gains were lost with discontinuation of practice (see Draganski \& May, 2008 for review). At least one additional follow-up assessment is needed to establish the durability of changes reported here.

Sixth, brain volume and changes therein may be affected by multiple genetic factors such as the ApoE\&4 allele (Moffat, Szekely,
Zonderman, Kabani, and Resnick, 2000), or by their interaction with vascular risk (Bender \& Raz, 2012; de Frias et al., 2007). Unfortunately, at the time of writing, we had no information about the genotypes of our participants, and thus were unable to examine the possibility of genetic risks contributing to the observed declines.

Finally, in this study, we used only one indicator of structural change, i.e. the regional brain volume. Future studies should address the possibility that training can affect other aspects of brain anatomy, including the local microstructure (e.g., as used in Lövdén et al., 2012) and vascular architecture.

\subsection{Conclusion}

In healthy adults, age-related shrinkage of selected brain regions is detectable within a period shorter than 6 months. Self-reported history of treated hypertension was associated with greater shrinkage. In at least one region, the cerebellum, intensive cognitive training was associated with slower shrinkage, although this apparent amelioration of brain change does not relate specifically to improvement in the targeted cognitive skills.

\section{Acknowledgments}

This project was supported by the Max Planck Society grant from the Innovation Fund, the German Federal Ministry for Education and Research (BMBF), the Deutsche Forschungsgemeinschaft, the Sofja Kovalevskaja Award administered by the Alexander von Humboldt Foundation (to ML), and by the grant R37 AG-011230 from the National Institute on Aging, USA (to NR).

\section{References}

Abrahamsson, T., Cathala, L., Matsui, K., Shigemoto, R., \& Digregorio, D. A. (2012) Thin dendrites of cerebellar interneurons confer sublinear synaptic integration and a gradient of short-term plasticity. Neuron, 73, 1159-1172 (Epub 2012 March 21).

Allen, J. S., Bruss, J., Mehta, S., Grabowski, T., Brown, C. K., \& Damasio, H. (2008) Effects of spatial transformation on regional brain volume estimates. $\begin{array}{llll}\text { Neuroimage, } & 42(2), & 535-547 . & \text { http://dx.doi.org/10.1016/ }\end{array}$ j.neuroimage.2008.05.047. (Epub 2008 Jun 3)

Andersen, B. B., Gundersen, H. J., \& Pakkenberg, B. (2003). Aging of the human cerebellum: A stereological study. Journal of Comparative Neurology, 466 $356-365$.

Arendt, T. (2003). Synaptic plasticity and cell cycle activation in neurons are alternative effector pathways: The 'Dr. Jekyll and Mr. Hyde concept' of Alzheimer's disease or the yin and yang of neuroplasticity. Progress in Neurobiology, 71, 83-248.

Balsters, J. H., Cussans, E., Diedrichsen, J., Phillips, K. A., Preuss, T. M., Rilling, J. K., et al. (2010). Evolution of the cerebellar cortex: The selective expansion of prefrontal-projecting cerebellar lobules. NeuroImage, 49, 2045-2052 (Epub 2009 October 24).

Balsters, J. H., \& Ramnani, N. (2011). Cerebellar plasticity and the automation of first-order rules. Journal of Neuroscience, 31, 2305-2312.

Bender, A. R., \& Raz, N. (2012). Age-related differences in memory and executive functions in healthy APOE \&4 carriers: The contribution of individual differences in prefrontal volumes and systolic blood pressure. Neuropsychologia, 50, 704-714.

Black, J. E., Sirevaag, A. M., \& Greenough, W. T. (1987). Complex experience promotes capillary formation in young rat visual cortex. Neuroscience Letters, 83 , 351-355.

Black, J. E., Isaacs, K. R., Anderson, B. J., Alcantara, A. A., \& Greenough, W. T. (1990) Learning causes synaptogenesis, whereas motor activity causes angiogenesis, in cerebellar cortex of adult rats. Proceedings of the National Academy of Sciences of the United States of America, 87, 5568-5572.

Bobinski, M., de Leon, M. J., Wegiel, J., Desanti, S., Convit, A., Saint Louis, L. A., et al. (2000). The histological validation of post mortem magnetic resonance imaging-determined hippocampal volume in Alzheimer's disease. Neuroscience, 95, 721-725.

Botez, M. I., Botez, T., Elie, R., \& Attig, E. (1989). Role of the cerebellum in complex human behavior. Italian Journal of Neurological Sciences, 10, 291-300.

Boyke, J., Driemeyer, J., Gaser, C., Büchel, C., \& May, A. (2008). Training-induced brain structure changes in the elderly. Journal of Neuroscience, 28, 7031-7035.

Cabeza, R., \& Nyberg, L. (2000). Imaging cognition II: An empirical review of 275 PET and fMRI studies. Journal of Cognitive Neuroscience, 12, 1-47. 
Cherbuin, N., Anstey, K. J., Réglade-Meslin, C., \& Sachdev, P. S. (2009). In vivo hippocampal measurement and memory: A comparison of manual tracing and automated segmentation in a large community-based sample. PLOS ONE, 4(4), e5265. http://dx.doi.org/10.1371/journal.pone.0005265 (Epub 2009 April 16).

Courchesne, E., Chisum, H. J., Townsend, J., Cowles, A., Covington, J., Egaas, B., et al. (2000). Normal brain development and aging: Quantitative analysis at in vivo MR imaging in healthy volunteers. Radiology, 216, 672-682.

de Frias, C. M., Bunce, D., Wahlin, A., Adolfsson, R., Sleegers, K., Cruts, M., et al. (2007). Cholesterol and triglycerides moderate the effect of apolipoprotein $\mathrm{E}$ on memory functioning in older adults. Journals of Gerontology. Series B Psychological Sciences and Social Sciences, 62, P112-P118.

Delisi, L. E., Sakuma, M., Tew, W., Kuschner, M., Hoff, A. L., \& Grimson, R. (1997). Schizophrenia as a chronic active brain process: A study of progressive brain structural change subsequent to the onset of schizophrenia. Journal of Psychiatric Research, 74, 129-140.

Diamond, A. (2000). Close interrelation of motor development and cognitive development and of the cerebellum and prefrontal cortex. Child Development, 71(1), 44-56.

Draganski, B., \& May, A. (2008). Training-induced structural changes in the adult human brain. Behavioural Brain Research, 192, 137-142 (Epub 2008 February 17).

Draganski, B., Gaser, C., Bush, V., Schuierer, G., Boghdan, U., \& May, A. (2004) Neuroplasticity: Changes in the gray matter introduced by training. Nature, 427, 311-312.

Draganski, B., Gaser, C., Kempermann, G., Kuhn, H. G., Winkler, J., Büchel, C., et al. (2006). Temporal and spatial dynamics of brain structure changes during extensive learning. Journal of Neuroscience, 26, 6314-6317.

Driemeyer, J., Boyke, J., Gaser, C., Büchel, C., \& May, A. (2008). Changes in gray matter induced by learning-revisited. PLOS ONE, 3, e2669.

Driscoll, I., Davatzikos, C., An, Y., Wu, X., Shen, D., Kraut, M., et al. (2009). Longitudinal pattern of regional brain volume change differentiates normal aging from MCI. Neurology, 72, 1906-1913.

Duning, T., Kloska, S., Steinsträter, O., Kugel, H., Heindel, W., \& Knecht, S. (2005) Dehydration confounds the assessment of brain atrophy. Neurology, 64 548-550.

Eckert, M. A., Keren, N. I., Roberts, D. R., Calhoun, V. D., \& Harris, K. C. (2010). Agerelated changes in processing speed: unique contributions of cerebellar and prefrontal cortex. Frontiers in Human Neuroscience, 4, 10. http://dx.doi.org/ 10.3389/neuro.09.010.2010.

Engvig, A., Fjell, A. M., Westlye, L. T., Moberget, T., Sundseth, Ø., Larsen, V. A., et al (2010). Effects of memory training on cortical thickness in the elderly. NeuroImage, 52, 1667-1676.

Engvig, A., Fjell, A. M., Westlye, L. T., Moberget, T., Sundseth, Ø., Larsen, V. A., et al. (2012). Memory training impacts short-term changes in aging white matter: A longitudinal diffusion tensor imaging study. Human Brain Mapping, 33, 2390-2406. http://dx.doi.org/10.1002/hbm.21370 (Epub 2011 August 5).

Erickson, K. I., Voss, M. W., Prakash, R. S., Basak, C., Szabo, A., Chaddock, L., et al. (2011). Exercise training increases size of hippocampus and improves memory. Proceedings of the National Academy of Sciences of the United States of America, 108, 3017-3022 (Epub 2011 January 31).

Eriksson, S. H., Free, S. L., Thom, M., Symms, M. R., Martinian, L., Duncan, J. S., et al. (2009). Quantitative grey matter histological measures do not correlate with grey matter probability values from in vivo MRI in the temporal lobe. Journal of Neuroscience Methods, 181(1), 111-118. http://dx.doi.org/10.1016/ j.jneumeth.2009.05.001 (Epub 2009 May 9).

Farkas, E., \& Luiten, P. G. (2001). Cerebral microvascular pathology in aging and Alzheimer's disease. Progress in Neurobiology, 64, 575-611.

Fjell, A. M., Walhovd, K. B., Fennema-Notestine, C., McEvoy, L. K., Hagler, D. J., Holland, D., et al. (2009). One-year brain atrophy evident in healthy aging. Journal of Neuroscience, 29, 15223-15231.

Groussard, M., La Joie, R., Rauchs, G., Landeau, B., Chételat, G., Viader, F., et al. (2010) When music and long-term memory interact: Effects of musical expertise on functional and structural plasticity in the hippocampus. PLOS ONE, 5, e13225. http://dx.doi.org/10.1371/journal.pone.0013225.

Hedden, T., \& Gabrieli, J. D. (2004). Insights into the ageing mind: a view from cognitive neuroscience. Nature Reviews Neuroscience, 5(2), 87-96.

Hoekzema, E., Carmona, S., Ramos-Quiroga, J. A., Barba, E., Bielsa, A., Tremols, V., et al. (2011). Training-induced neuroanatomical plasticity in ADHD: A tensorbased morphometric study. Human Brain Mapping, 32, 1741-1749. http:/ dx.doi.org/10.1002/hbm.21143 (Epub 2011 March 1).

Isaacs, K. R., Anderson, B. J., Alcantara, A. A., Black, J. E., \& Greenough, W. T. (1992) Exercise and the brain: Angiogenesis in the adult rat cerebellum after vigorous physical activity and motor skill learning. Journal of Cerebral Blood Flow and Metabolism, 12, 110-119.

Ito, M. (2002). Historical review of the significance of the cerebellum and the role of Purkinje cells in motor learning. Annals of the New York Academy of Sciences, 978 273-288.

Jack, C. R., Jr., Twomey, C. K., Zinsmeister, A. R., Sharbrough, F. W., Petersen, R. C., \& Cascino, G. D. (1989). Anterior temporal lobes and hippocampal formations: Normative volumetric measurements from MR images in young adults. Radiology, 172, 549-554.

Jennings, J. R., Mendelson, D. N., Muldoon, M. F., Ryan, C. M., Gianaros, P. J., Raz, N. et al. (2012). Regional grey matter shrinks in hypertensive individuals despite successful lowering of blood pressure. Journal of Human Hypertension, 26 , 295-305. http://dx.doi.org/10.1038/jhh.2011.31 (Epub 2011 April 14).
Kemper, T. L. (1994). Neuroanatomical and neuropathological changes during aging and in dementia. In M. L. Albert \& E. J. E. Knoepfel (Eds.), Clinical neurology of aging (2nd ed., pp. 3-67). New York: Oxford University Press.

Kleim, J. A., Markham, J. A., Vij, K., Freese, J. L., Ballard, D. H., \& Greenough, W. T. (2007). Motor learning induces astrocytic hypertrophy in the cerebellar cortex. Behavioural Brain Research, 178, 244-249 (Epub 2007 January 25).

Korf, E. S., White, L. R., Scheltens, P., \& Launer, L. J. (2004). Midlife blood pressure and the risk of hippocampal atrophy: The Honolulu Asia Aging Study. Hypertension, 44, 29-34 (Epub 2004 May 24).

Landi, S. M., Baguear, F., \& Della-Maggiore, V. (2011). One week of motor adaptation induces structural changes in primary motor cortex that predict long-term memory one year later. Journal of Neuroscience, 31, 11808-11813.

Lenroot, R. K., \& Giedd, J. N. (2006). Brain development in children and adolescents: insights from anatomical magnetic resonance imaging. Neuroscience and Biobehavioral Reviews, 30(6), 718-729 (Epub 2006 Aug 2).

Lövdén, M., Bäckman, L., Lindenberger, U., Schaefer, S., \& Schmiedek, F. (2010a). A theoretical framework for the study of adult cognitive plasticity. Psychological Bulletin, 136, 659-676.

Lövdén, M., Bodammer, N. C., Kühn, S., Kaufmann, J., Schütze, H., Tempelmann, C., et al. (2010b). Experience-dependent plasticity of white-matter microstructure extends into old age. Neuropsychologia, 48, 3878-3883 (Epub 2010 September 15).

Lövdén, M., Schaefer, S., Noack, H., Bodammer, N. C., Kühn, S., Heinze, H. J., et al. (2012). Spatial navigation training protects the hippocampus against agerelated changes during early and late adulthood. Neurobiology of Aging, 33, 620.e9-620.e22 (Epub 2011 April 16).

Mackey, A. P., Whitaker, K. J., \& Bunge, S. A. (2012). Experience-dependent plasticity in white matter microstructure: Reasoning training alters structural connectivity. Frontiers in Neuroanatomy, 6, 32 (Epub 2012 August 22).

Maguire, E. A., Gadian, D. G., Johnsrude, I. S., Good, C. D., Ashburner, J., Frackowiak, R. S., et al. (2000). Navigation-related structural change in the hippocampi of taxi drivers. Proceedings of the National Academy of Sciences of the United States of America, 97, 4398-4403.

Mårtensson, J., Eriksson, J., Bodammer, N. C., Lindgren, M., Johansson, M., Nyberg, L., et al. (2012). Growth of language-related brain areas after foreign language learning. NeuroImage, 63, 240-244 (Epub 2012 June 29).

May, A. (2011). Experience-dependent structural plasticity in the adult human brain. TICS, $15,475-482$.

McArdle, J. J. (2009). Latent variable modeling of differences and changes with longitudinal data. Annual Review of Psychology, 60, 577-605.

McArdle, J. J., \& Nesselroade, J. R. (1994). Using multivariate data to structure developmental change. In S. H. Cohen \& H. W. Reese (Eds.), Life-span developmental psychology: Methodological contributions (pp. 223-267). Hillsdale, NJ: Lawrence Erlbaum Associates.

McArdle, J. J., Hamagami, F., Jones, K., Jolesz, F., Kikinis, R., Spiro, R., et al. (2004). Structural modeling of dynamic changes in memory and brain structure using longitudinal data from the Normative Aging Study. Journals of Gerontology. Series B, Psychological Sciences and Social Sciences, 59, P294-P304.

Moffat, S. D., Szekely, C. A., Zonderman, A. B., Kabani, N. J., \& Resnick, S. M. (2000). Longitudinal change in hippocampal volume as a function of apolipoprotein $\mathrm{E}$ genotype. Neurology, 55(1), 134-136.

Mora, F., Segovia, G., \& del Arco, A. (2007). Aging, plasticity and environmental enrichment: Structural changes and neurotransmitter dynamics in several areas of the brain. Brain Research Reviews, 55, 78-88 (Epub 2007 April 13).

Murphy, E. A., Holland, D., Donohue, M., McEvoy, L. K., Hagler, D. J., Jr., Dale, A. M., et al. (2010). Six-month atrophy in MTL structures is associated with subsequent memory decline in elderly controls. NeuroImage, 53, 1310-1317 (Epub 2010 July 13).

Pereira, A. C., Huddleston, D. E., Brickman, A. M., Sosunov, A. A., Hen, R., McKhann, G. M., et al. (2007). An in vivo correlate of exercise-induced neurogenesis in the adult dentate gyrus. Proceedings of the National Academy of Sciences of the United States of America, 104, 5638-5643.

Pfefferbaum, A., Sullivan, E. V., Rosenbloom, M. J., Mathalon, D. H., \& Lim, K. O. (1998). A controlled study of cortical gray matter and ventricular changes in alcoholic men over a 5-year interval. Archives of General Psychiatry, 55, 905-912.

Raz, N. (2000). Aging of the brain and its impact on cognitive performance: Integration of structural and functional findings. In F. I. M. Craik \& T. A. Salthouse (Eds.), Handbook of aging and cognition - II (pp. 1-90). Mahwah, NJ: Erlbaum.

Raz, N., \& Rodrigue, K. (2006). Differential aging of the brain: Patterns, cognitive correlates and modifiers. Neuroscience and Biobehavioral Reviews, 30, 730-748.

Raz, N., \& Kennedy, K. M. (2009). A systems approach to age-related change: Neuroanatomic changes, their modifiers, and cognitive correlates. In W. Jagust \& M. D’Esposito (Eds.), Imaging the aging brain (pp. 43-70). New York, NY: Oxford University Press.

Raz, N., Rodrigue, K. M., Kennedy, K. M., Dahle, C., Head, D., \& Acker, J. D. (2003a). Differential age-related changes in the regional metencephalic volumes in humans: A five-year follow-up. Neuroscience Letters, 349, 163-166.

Raz, N., Rodrigue, K. M., Kennedy, K. M., Head, D., Gunning-Dixon, F. M., \& Acker, J. D. (2003b). Differential aging of the human striatum: Longitudinal evidence. AJNR, $24,1849-1856$.

Raz, N., Gunning-Dixon, F., Head, D., Williamson, A., Rodrigue, K., \& Acker, J. D. (2004). Aging, sexual dimorphism, and hemispheric asymmetry of the cerebral cortex: Replicability of regional differences in volume. Neurobiology of Aging, 25, 377-396. 
180

N. Raz et al./Brain and Cognition 82 (2013) 171-180

Raz, N., Lindenberger, U., Rodrigue, K. M., Kennedy, K. M., Head, D., Williamson, A., et al. (2005). Regional brain changes in aging healthy adults: General trends, individual differences, and modifiers. Cerebral Cortex, 15, 1676-1689.

Raz, N., Rodrigue, K. M., Kennedy, K. M., \& Acker, J. D. (2007). Vascular health and longitudinal changes in brain and cognition in middle-aged and older adults. Neuropsychology, 21, 149-157.

Raz, N., Lindenberger, U., Ghisletta, P., Rodrigue, K. M., Kennedy, K. M., \& Acker, J. D. (2008). Neuroanatomical correlates of fluid intelligence in healthy adults and persons with vascular risk factors. Cerebral Cortex, 18, 718-726 (2007 July 5; [Epub ahead of print]).

Raz, N., Ghisletta, P., Rodrigue, K. M., Kennedy, K. M., \& Lindenberger, U. (2010). Trajectories of brain aging in middle-aged and older adults: Regional and individual differences. NeuroImage, 51, 501-511 (Epub 2010 March 16).

Resnick, S. M., Pham, D. L., Kraut, M. A., Zonderman, A. B., \& Davatzikos, C. (2003). Longitudinal magnetic resonance imaging studies of older adults: A shrinking brain. Journal of Neuroscience, 23, 3295-3301.

Riddle, D. R., Sonntag, W. E., \& Lichtenwalner, R. J. (2003). Microvascular plasticity in aging. Ageing Research Reviews, 2, 149-168.

Roche, R. A., Mullally, S. L., McNulty, J. P., Hayden, J., Brennan, P., Doherty, C. P., et al. (2009). Prolonged rote learning produces delayed memory facilitation and metabolic changes in the hippocampus of the ageing human brain. BMC Neuroscience, 10, 136. http://dx.doi.org/10.1186/1471-2202-10-136.

Rochefort, C., Arabo, A., André, M., Poucet, B., Save, E., \& Rondi-Reig, L. (2011). Cerebellum shapes hippocampal spatial code. Science, 334, 385-389.

Rodrigue, K. M., \& Raz, N. (2004). Shrinkage of the entorhinal cortex over five years predicts memory performance in healthy adults. The Journal of Neuroscience, 24 956-963.

Rogosa, D. R., \& Willett, J. B. (1985). Understanding correlates of change by modeling individual differences in growth. Psychometrika, 50, 203-228.

Rusinek, H., De Santi, S., Frid, D., Tsui, W. H., Tarshish, C. Y., Convit, A., et al. (2003). Regional brain atrophy rate predicts future cognitive decline: 6-year longitudinal MR imaging study of normal aging. Radiology, 229, 691-696.

Sagi, Y., Tavor, I., Hofstetter, S., Tzur-Moryosef, S., Blumenfeld-Katzir, T., \& Assaf, Y. (2012). Learning in the fast lane: New insights into neuroplasticity. Neuron, 73 , 1195-1203 (Epub 2012 March 21).
Salmi, J., Pallesen, K. J., Neuvonen, T., Brattico, E., Korvenoja, A., Salonen, O., et al. (2010). Cognitive and motor loops of the human cerebro-cerebellar system. Journal of Cognitive Neuroscience, 22, 2663-2676.

Salerno, J. A., Murphy, D. G., Horwitz, B., DeCarli, C., Haxby, J. V., Rapoport, S. I., et al. (1992). Brain atrophy in hypertension. A volumetric magnetic resonance imaging study. Hypertension, 20(3), 340-348.

Scahill, R. I., Frost, C., Jenkins, R., Whitwell, J. L., Rossor, M. N., \& Fox, N. C. (2003). A longitudinal study of brain volume changes in normal aging using serial registered magnetic resonance imaging. Archives of Neurology, 60, 989-994.

Schmidt-Wilcke, T., Rosengarth, K., Luerding, R., Bogdahn, U., \& Greenlee, M. W. (2010). Distinct patterns of functional and structural neuroplasticity associated with learning Morse code. Neuroimage, 51, 1234-1241 (Epub 2010 March 24).

Schmiedek, F., Lövdén, M., \& Lindenberger, U. (2010). Hundred days of cognitive training enhance broad cognitive abilities in adulthood: Findings from the COGITO study. Frontiers in Aging Neuroscience, 2. http://dx.doi.org/10.3389/ fnagi.2010.00027.

Shen, L., Saykin, A. J., Kim, S., Firpi, H. A., West, J. D., Risacher, S. L., et al. (2010). Comparison of manual and automated determination of hippocampal volumes in MCI and early AD. Brain Imaging and Behavior, 4(1), 86-95. http://dx.doi.org/ 10.1007/s11682-010-9088-X.

Shrout, P. E., \& Fleiss, J. L. (1979). Intraclass correlations: uses in assessing rater reliability. Psychol Bull, 86(2), 420-428.

Strassburger, T. L., Lee, H. C., Daly, E. M., Szczepanik, J., Krasuski, J. S., Mentis, M. J., et al. (1997). Interactive effects of age and hypertension on volumes of brain structures. Stroke, 28, 1410-1417.

Strick, P. L., Dum, R. P., \& Fiez, J. A. (2009). Cerebellum and nonmotor function. Annual Review of Neuroscience, 32, 413-434.

Takeuchi, H., Sekiguchi, A., Taki, Y., Yokoyama, S., Yomogida, Y., Komuro, N., et al. (2010). Training of working memory impacts structural connectivity. Journal of Neuroscience, 30, 3297-3303.

Thomas, C., \& Baker, C. I. (2012). Teaching an adult brain new tricks: A critical review of evidence for training-dependent structural plasticity in humans. Neuroimage, 59, 3389-3397 (March 30. [Epub ahead of print]).

Wenger, E., Schaefer, S., Noack, H., Kühn, S., Mårtensson, J., Heinze, H.-J., et al. (2012). Cortical thickness changes following spatial navigation training in adulthood and aging. Neurolmage, 59, 3389-3397. 